\title{
Research and optimization of a new design of output window for high power microwave tubes
}

\author{
Anatoly Galdetskiy ${ }^{*}$ and Alexander Savin \\ JSC "RPC "Istok" named after Shokin", Fryazino, 141190, Russian Federation
}

\begin{abstract}
A design of an output window integrated with an output resonator of the high-power klystron is proposed. The results of a theoretical study of the frequency response of an integrated window are presented. Calculation of the electric field in critical areas of the optimized design showed that the losses in the vacuum-tight dielectric are much lower, and the tangential components of the field on its surface, which are the source of breakdowns, are lower or comparable with similar parameters of traditional pillbox windows.
\end{abstract}

\section{Introduction}

One of the key problems in the development of S-band klystrons with pulse power $P_{\text {imp }}=5-150 \mathrm{MW}$ is the design of vacuum-tight windows, since in order to prevent microwave breakdown it is necessary to ensure low values of the electric field in the window cavity, on the dielectric surface and in the brazing area. Also, it is necessary to ensure good matching and a low level of microwave losses in the working frequency band (usually fairly narrow, less than $1 \%$ ).

Traditionally, to solve these problems, pillbox windows are used, operating on the $\mathrm{TE}_{11}$, $\mathrm{TM}_{11}$ and on mixed $\mathrm{TE}_{11}+\mathrm{TM}_{11}$ modes $[1,2]$. Moreover, any pillbox window creates an additional discontinuity in the output waveguide, the reflection of which is required to be compensated by matching elements that usually reduce the electric strength.

To increase reliability of the window at operation at large powers in klystron, we propose embedding the vacuum-tight septum to the output resonator and positioning it in a region with a minimum electric field (Fig. 1). In this way, additional perturbation by the window in the output waveguide is absorbed by coupling slots discontinuity and the probability of microwave breakdown and overheating of the vacuum-tight septum due to dielectric losses is reduced.

The work is devoted to the study and optimization of electrodynamic parameters of a new type of window, integrated with the output resonator and designed to work with increased power level.

\section{Object and methods of research}

We consider design of output window integrated with the output resonator of high-power klystron (Fig. 1).

* Corresponding author: galdetskiy@istokmw.ru 


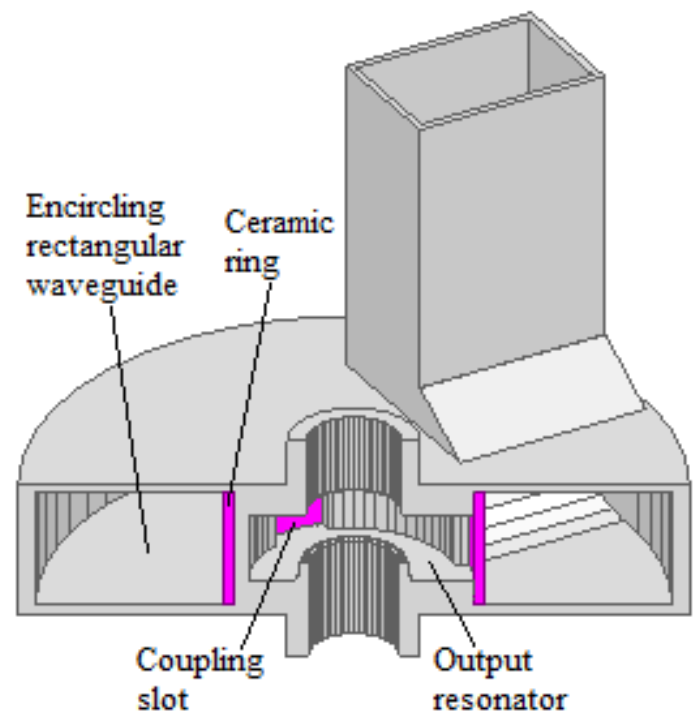

a)

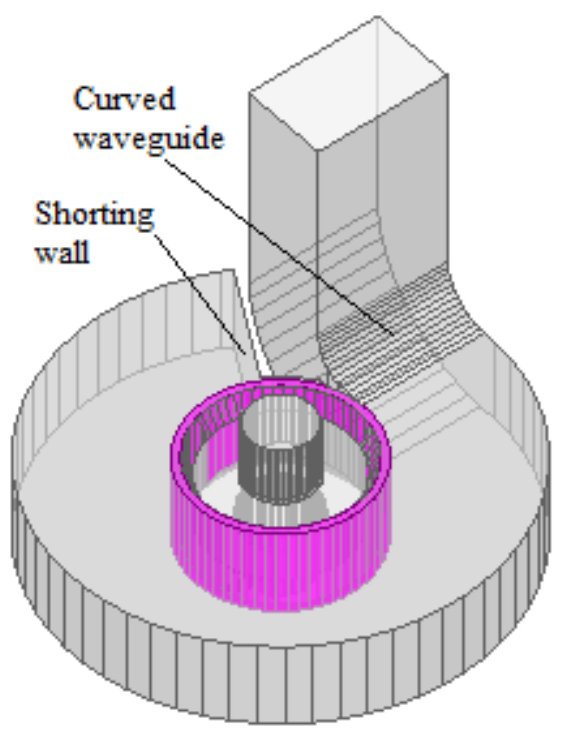

b)

Fig. 1. High-power klystron output window integrated with the output resonator. a) - device appearance (sliced), b) - vacuum volume.

The integrated output window contains a rectangular waveguide shorted on one end operating on the $\mathrm{TE}_{10}$ mode and encircling the output cavity (Fig. 1). Inductive coupling slots are located in a common narrow wall and summ up the power coming from each slot to the output of a encircling waveguide and provide the required loaded Q-factor of the output cavity.

Ring vacuum-tight ceramic septum is located in the region with minimum electric field to reduce dielectric losses - inside the output cavity near the outer wall or in the encircling waveguide near its narrow wall. In the last case, part of the ring passes through the section, which is below-cutoff waveguide for the working wave type and provides isolation between ends of circular waveguide. The transport of microwave energy flow to the load is carried out using a segment of a curved waveguide connected to the output of a encircling waveguide.

Thus, the proposed design combines in one device a wave type converter and a vacuumtight window. This eliminates additional discontinuities and tuning elements in the output waveguide of the high-power klystron.

The proposed design was synthesized by successive single-, two-criteria optimization of it's geometry dimensions. The calculation of the electrodynamic characteristics was carried out by the finite element solver in the ANSYS HFSS program.

\section{Results of reseach}

For effective summation of partial powers coming from each slot they must be separated by distance equal to $\lambda_{\mathrm{w}}-$ single wavelength in waveguide. At design optimization for operation in S-band it is possible to place only three coupling slots in common narrow wall of the output resonator and the encircling rectangular waveguide (Fig. 2a).

The coupling between output cavity and the waveguide was adjusted to provide required loaded Q-factor equal to 26 by changing the width, shape of the edges, and the number of slots. To equalize the fields in different coupling slots, the wavelength $\lambda_{\mathrm{w}}$ in the encircling 
waveguide was tuned at fixed slots positions by varying the radius of waveguide outer narrow wall.

The distribution of the electric field in the integrated window (Fig. 2a) and the azimuthal dependence of the electric field amplitude on the center line of the encircling rectangular waveguide (fig. 2 b) demonstrate the process of formation of a traveling wave at waveguide output.

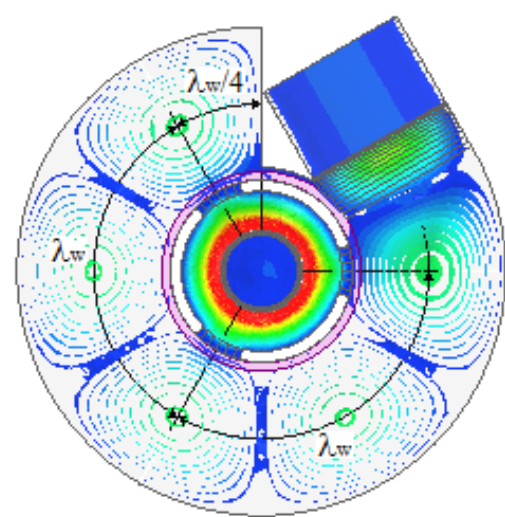

a)

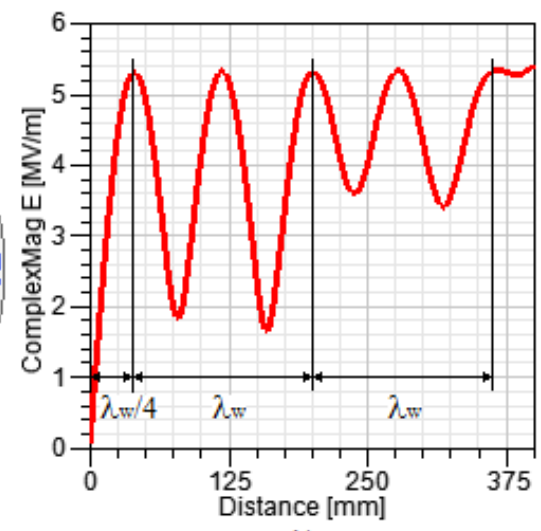

b)

Fig. 2. a) - electric field distribution in the integrated window at the center frequency, b) the complex amplitude of the electric field on the center line of the encircling rectangular waveguide vs. azimuthal coordinate at output power $P=40 \mathrm{MW}$.

We calculated frequency dependence of the power in output waveguide when resonator is excited by fixed rf-current in the cavity gap. Current amplitude is set to take power from the current source equal to $40 \mathrm{MW}$ at the center frequency. At loaded Q-factor tuned to $Q_{\mathrm{L}}=27, Q_{\mathrm{L}}=48, Q_{\mathrm{L}}=58$ the frequency recponses are shown in the Fig. 3a-c correspondingly. In all cases the circuit efficiency of the integrated window was very close to 1. Thus, it is demonstrated that the proposed output window design is capable the implementation of various loaded Q-factors of the output cavity and provides the required electrodynamic characteristics.

Main reasons for the unreliable operation of windows in high-power klystrons are the presence of significant tangential components of the electric field on the surface of the ceramic septum, large field in the brazing area, significant electric field in the vacuum or gas-filled part, leading to breakdown, as well as overheating of the ceramic due to dielectric loss [1]. For this reason the maximum value of the tangential electric field for aluminabased ceramics is $\sim 8 \mathrm{MV} / \mathrm{m}$ and for the electric field in the waveguide $\sim 12 \mathrm{MV} / \mathrm{m}[1]$.

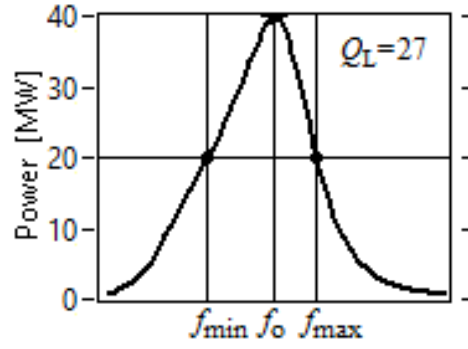

a)

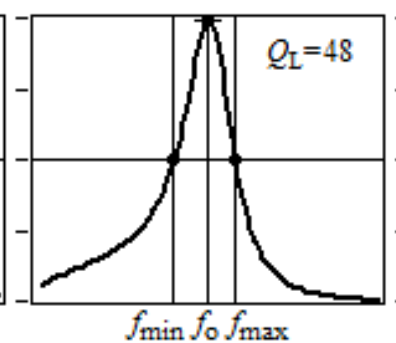

b)

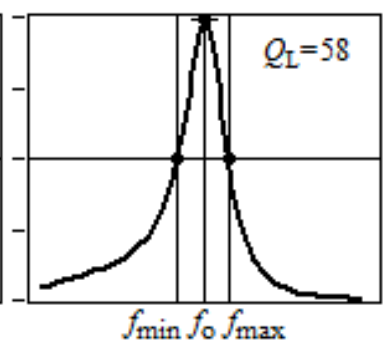

c)

Fig. 3. Frequency dependences of the output power at cavity excitation by fixed rf-current and loaded Q-factors a) $\left.\left.-Q_{\mathrm{L}}=27, \mathrm{~b}\right)-Q_{\mathrm{L}}=48, \mathrm{c}\right)-Q_{\mathrm{L}}=58$. 
To estimate the limiting energy characteristics of the proposed integrated output window the maximal tangential components of the electric field were calculated on the inner and outer surfaces of the ceramic ring close to coupling slots at $P=40 \mathrm{MW}$ for the operating frequency in S-band. Similar calculations were also made for traditional pillbox windows designed to operate at the same frequency. Table 1 shows parameters which are critical for the microwave breakdown and ceramics overheating for traditional pillbox windows and proposed integrated window.

Table 1. Energy parameters at $P=40 \mathrm{MW}$ at the center frequency.

\begin{tabular}{|c|c|c|c|c|c|c|}
\hline Window type & $\begin{array}{c}\text { Working } \\
\text { wave type }\end{array}$ & $\begin{array}{c}\boldsymbol{E}_{\mathbf{m a x}}, \\
\mathbf{M V} / \mathbf{m}\end{array}$ & $\begin{array}{c}\boldsymbol{E}_{\boldsymbol{\tau}} \text { at center } \\
\text { of ceramics, } \\
\mathbf{M V} / \mathbf{m}\end{array}$ & $\begin{array}{c}\boldsymbol{E}_{\boldsymbol{\tau}} \text { at edge } \\
\text { of ceramics, } \\
\mathbf{M V} / \mathbf{m}\end{array}$ & $\begin{array}{c}\boldsymbol{E}_{\mathbf{m a x}} \text { in output } \\
\text { waveguide, } \\
\mathbf{M V} / \mathbf{m}\end{array}$ & $\begin{array}{c}\text { Losses in } \\
\text { ceramics, } \\
\mathbf{k W}\end{array}$ \\
\hline Pillbox & $\mathrm{TE}_{11}$ & 10.23 & 3.86 & 2.04 & 6 & 8.27 \\
\hline Pillbox & $\mathrm{TM}_{11}$ & 13.55 & 4.09 & 3.29 & 4.28 & 27.48 \\
\hline Pillbox & $\mathrm{TE}_{11}+\mathrm{TM}_{11}$ & 8.89 & 4.33 & 0.36 & 4.84 & 17.95 \\
\hline New window & $\mathrm{TE}_{10}$ & 5.5 & 3.5 & 2 & 5.5 & 5.45 \\
\hline
\end{tabular}

An analysis of the electric fields shown in the table demonstrates that proposed integrated window has a 1.5 -fold margin for output power at $P=40 \mathrm{MW}$, and there are no such margins for traditional pillbox windows or even the electric fields exceed the limiting values. Losses in ceramics of new window are also significantly less than similar losses in traditional pillbox windows.

\section{Conclusion}

As a result of the study, it was found that the proposed window, integrated with the output resonator of high-power klystron provides the required frequency characteristics when working in S-band at output power $P=40 \mathrm{MW}$, and has a significant margin on breakdown strength.

The calculated losses in the ceramics, leading to its heating, are only $5.45 \mathrm{~kW}$ (pulsed), which is $\sim 3$ times less than the similar losses in the pillbox window, operating in a mixed mode and having the highest power capability. In addition, the proposed output window does not require additional tuning to provide good matching, and the absence of reflections in the waveguide significantly increases its breakdown strength.

A further doubling of the maximal output power in the proposed design is possible by replacing the shorting wall with a second output waveguide with a corresponding decrease of the field amplitude in the waveguides.

\section{References}

1. B.V. Prokofiev, M.A. Martynenko A Simple Pillbox-Type Mixed-Mode Window for High Power Microwave Devices // in 2017 Eighteenth International Vacuum Electronics Conference (IVEC), pp. 1-2 (2017)

2. S.Yu. Kazakov A new traveling-wave mixed-mode RF window with a low electric field in ceramic-metal brazing area // KEK preprint 98-120, pp. 1-4 (1998) 